\title{
Die Geschichte zu dieser Arbeit
}

Dass diese Arbeit entstanden ist und ich Professorin werden konnte, habe ich vor allem Prof. Dr. Werner Knapp und Prof. Dr. Bernt Ahrenholz zu verdanken.

Im November 2009 hielt ich gemeinsam mit meinem Doktorvater Werner Knapp in Augsburg einen Vortrag auf dem alljährlich stattfindenden DaZ-Workshop. Werner Knapp erwähnte in diesem Zusammenhang, dass ich in Kürze meine Dissertation einreichen würde. In der darauffolgenden Woche erreichte mich eine Email von Bernt Ahrenholz, der auch in Augsburg gewesen war und der mir eine Habilitationsstelle in Jena anbot. Da ich eigentlich als Lehrerin im Schuldienst tätig sein wollte und bereits eine Zusage für einen Referendariatsplatz erhalten hatte, dachte ich, zur tiefen Irritation meines Doktorvaters, zunächst daran, das Stellenangebot auszuschlagen. Ich bin Werner Knapp heute auch dafür sehr dankbar, dass er mich dann doch davon überzeugt hat, die Stelle in Jena anzutreten, die den institutionellen und fachlichen Rahmen für die Entstehung dieser Arbeit geschaffen hat.

Während meiner Zeit am Lehrstuhl von Bernt Ahrenholz an der FriedrichSchiller-Universität (FSU) Jena durfte ich viel von ihm und anderen Kollegen und Kolleginnen lernen, sodass ich diese Zeit stets als fachlich inspirierend empfunden habe. Gerne erinnere ich mich beispielsweise an die regelmäßigen, motivierenden „Habil.-Tandems“ mit Dr. Eva Schmucker.

Bernt Ahrenholz hat mein Habilitationsprojekt bis zum Abschluss einlässlich begleitet. Im späten Frühjahr 2018 konnte ich die Monographie schließlich an der FSU als Habilitationsschrift zur Begutachtung einreichen. Nach Durchsicht der Arbeit hat sich Bernt Ahrenholz dann dafür eingesetzt, dass die Arbeit in die Reihe „DaZ-Forschung“ bei De Gruyter aufgenommen worden ist. Über diese Wertschätzung von seiner Seite und den drei weiteren Herausgeberinnen der Reihe habe ich mich sehr gefreut. Im November 2019, noch bevor er das Gutachten für meine Habilitationsschrift fertigstellen konnte, ist Bernt Ahrenholz einer schweren Krankheit erlegen. Ich bedaure es sehr, dass er so auch das Erscheinen dieser Monographie nicht mehr miterleben durfte. Bernt Ahrenholz ist diese Arbeit gewidmet.

Prof. Dr. Christine Czinglar, der Nachfolgerin von Bernt Ahrenholz an der FSU, bin ich dankbar dafür, dass sie die Begutachtung der Arbeit nach seinem Tod übernommen hat und ebenso dafür, dass sie mich mit wertvoller, konstruktiver Kritik bei der Erstellung der hier vorliegenden Druckfassung unterstützt hat. Ein weiterer Dank gilt Prof. Dr. Udo Ohm und Prof. Dr. Iris Winkler für ihre Bereitschaft, die Habilitationsschrift als weitere Kommissionsmitglieder zu lesen und zu begutachten. 
Verortet war und ist mein Habilitationsprojekt nicht nur an der FSU in Jena, sondern auch im Projekt „Fachunterricht und Deutsch als Zweitsprache“, das Bernt Ahrenholz geleitet hat. Durch diese Anbindung haben sich für mein Habilitationsprojekt zahlreiche Synergieeffekte ergeben: beispielsweise über die Möglichkeit, im Projekt entwickelte oder bereitgestellte Tools (wie den sprachbiographischen Fragebogen oder den von Prof. Dr. Wilhelm Grießhaber bereitgestellten C-Test) für mein Habilitationsprojekt nutzen zu können, aber auch durch die Zusammenarbeit mit meiner Kollegin Dr. Diana Maak im Zuge der Datenerhebungen für das Projekt „Fachunterricht und Deutsch als Zweitsprache“.

Dankbar bin ich ferner Prof. Dr. Michael Ewig von der Pädagogischen Hochschule Weingarten für die Bereitstellung eines Videoclips mit einem Schulversuch als Impuls für die Datenerhebung, den Hilfskräften Franziska Kubald und Julia Exner für Unterstützung bei der Transkription der Versuchsprotokolle, Marc Reznicek für eine Beratung bezüglich des Datenworkflows und die Überführung der Word-Transkripte ins xls-Format, meiner Sekretärin Stephanie Smolik für ihre Unterstützung bei der Formatierung für die Drucklegung der Arbeit sowie Julie Miess und Anne Rudolph für die angenehme Zusammenarbeit und die professionelle Unterstützung seitens des Verlags.

2013 hat mich ein Ruf an die Europa-Universität Flensburg erreicht, zu einem Zeitpunkt, als die Habilitationsschrift erst im Entstehen war. Ich habe es als herausfordernd empfunden, für dieses große Projekt ,neben' der Professur die erforderliche Kraft aufzubringen und die nötige Ruhe zu finden. Ohne ein Forschungsfreisemester im Winter 2017/2018 hätte ich es wohl kaum geschafft, die Arbeit tatsächlich fertigzustellen. Verbracht habe ich dieses Freisemester in Valencia. Den Kolleginnen der „Departament de Filologia Anglesa i Alemanya“ der Universitat de València, insbesondere Prof. Dr. Ana Rosa Calero Valera und Prof. Dr. Brigitte Jirku, danke ich für ihre Gastfreundschaft und für die Bereitstellung ihres Büros. Die Zeit in Valencia war ungemein produktiv und zugleich schön. Begleitet hat mich dorthin auch mein Mann Prof. Dr. Günter Helmes. Wie oft er nun schon von der Aussicht auf einen Abschluss meines Habilitationsprojektes hören musste, weiß er vermutlich selbst nicht mehr. Für all die Unterstützung, den Zuspruch, aber auch für die nötige Zerstreuung, die er mir in diesen Jahren zuteil hat werden lassen, danke ich ihm von Herzen.

Schule und Unterricht sind mir ein aufrichtiges Anliegen. Deshalb war es mir wichtig, mein Habilitationsprojekt anwendungsorientiert und empirisch auszurichten und auf schulisches Lernen $\mathrm{zu}$ beziehen. Schlussendlich waren an diesem Projekt drei Schulen, fünf Biologielehrkräfte und 173 Schülerinnen und Schüler beteiligt. Ihre Daten bilden das Fundament dieser Arbeit; ohne ihr Mit- 
wirken und ihre Unterstützung hätte das Projekt nicht realisiert werden können. Obwohl ich sie aus Datenschutzgründen namentlich nicht nennen kann, möchte ich dennoch ein herzliches Dankeschön an sie richten. 
